\title{
Hindered Rotation in Methyl Alcohol with Note on Ethyl Alcohol
}

\author{
J. O. HALFORD \\ Department of Chemistry, University of Michigan, Ann Arbor, Michigan
}

(Received August 24, 1949)

\begin{abstract}
The potential barrier to the internal rotation in methyl alcohol is recalculated from the entropy with the aid of new molecular dimensions generously provided by Burkhard and Dennison. The barrier calculation is examined for temperature dependence and checked for reliability by recalculation with the Clapeyron equation substituted for parts of the data. The result is $1600 \pm 700 \mathrm{cal} . / \mathrm{mole}$, which includes the new spectroscopic barrier of Burkhard and Dennison at $326 \mathrm{~cm}^{-1}$. Within the range covered by the result, a residual entropy up to $0.50 \mathrm{cal} . / \mathrm{mole}$ deg. is possible, and the retention of $0.46 \mathrm{e} . \mathrm{u}$. by the crystal is consistent with a reasonable simple assumption about the distribution of hydrogen bonds.

It is suggested, by analogy, that the barrier to the hydroxyl group rotation in ethyl alcohol should be about $1000 \mathrm{cal} . / \mathrm{mole}$.
\end{abstract}

$T$ $\mathrm{HE}$ height of the potential barrier restricting the internal rotation in methyl alcohol has now been evaluated from the entropy, the heat capacity at ordinary temperatures, the infra-red absorption spectrum and micro-wave data.

The most reliable published value from the entropy is near $2500 \mathrm{cal} . /$ mole. ${ }^{1,2}\left(870 \mathrm{~cm}^{-1}\right)$. A much lower result (1350) by French and Rasmussen ${ }^{3}$ is not acceptable because of the use of a heat of vaporization at room temperature which cannot be aligned in any reasonable way with the accurately known heat of vaporization at the boiling point. A higher barrier (3400) obtained by Crawford $^{4}$ from essentially the same data, appears to be the result of an erroneous calculation of the barrier from the entropy of the motion.

Barrier evaluations from the vapor heat capacity are very recent. From data obtained by means of a heat conductivity method, Eucken and Franck ${ }^{5}$ found 1800 cal./mole. Rowlinson, ${ }^{6}$ however, employed the velocity of supersonic waves to obtain a result near $1300 \mathrm{cal}$./ mole.

All of the above evaluations are based upon essentially the same molecular model and the same normal frequencies for the remaining internal degrees of freedom. Since certain features of the model are gratuitously assumed, this is a possible source of error common to all the evaluations from thermal data. There is a secondary source of error, the effect of deviation from ideal gas behavior, which could be appreciable. The vapor heat capacities have been used over a range of temperatures because the barrier height is a double valued function of heat capacity in which the choice between the alternatives is based upon the temperature variation. None of the evaluations from the entropy, however, has been made over a range of temperatures to see if a single

\footnotetext{
${ }^{1}$ J. O. Halford and B. Pecherer, J. Chem. Phys. 6, 571 (1938)

2 K. S. Pitzer, J. Am. Chem. Soc. 70, 2140 (1948).

${ }^{3}$ F. A. French and R. S. Rasmussen, J. Chem. Phys. 14, 389 (1946).

4 B. L. Crawford, Jr., J. Chem. Phys. 8, 744 (1940).

- A. Eucken and E. U. Franck, Zeits. f. Elektrochemie 52, 195 (1948).

${ }^{6} \mathrm{~J}$. S. Rowlinson, Nature 162, 820 (1948).
}

barrier height is consistent with the data at different temperatures.

Koehler and Dennison ${ }^{7}$ have used the infra-red absorption data of Borden and Barker ${ }^{8}$ and of Lawson and Randall ${ }^{7}$ to make an estimate of the barrier height. When calculations of line spacings based upon a preliminary barrier of $770 \mathrm{~cm}^{-1}(2200 \mathrm{cal} . / \mathrm{mole})$ were compared with the data, the estimate was revised downward to $450 \mathrm{~cm}^{-1}$ (1300 cal./mole).

Burkhard and Dennison ${ }^{9}$ have extended the infrared observations to lower frequencies, with a resulting barrier estimate of $300 \mathrm{~cm}^{-1}$, and have calculated an accurate barrier, $326 \mathrm{~cm}^{-1}$ (932 cal./mole), from microwave data. Thus the correct barrier is considerably less than any value derived from thermal data, showing that some serious error has been made in the calculation of entropy and heat capacity. In addition to the barrier height, Burkhard and Dennison have evaluated the moments of inertia from the data, including the value $1.01 \times 10^{-40} \mathrm{~g} \mathrm{~cm}^{2}$ as a close approximation to the moment of the $\mathrm{OH}$ group about an axis through the center of gravity of the molecule parallel to the symmetry axis of the methyl group. This is much lower than the value used in any of the previous barrier determinations, and corresponds to a $\mathrm{COH}$ angle in the neighborhood of $125^{\circ}$. Although, according to Professor Dennison, this value is still preliminary and, therefore subject to possible change upon further investigation, it will be used to obtain limiting barrier estimates.

In the present paper, the entropy of the internal rotation is recalculated with the new molecular dimensions, and barrier estimates are made from the data and a selected equation of state at a number of temperatures from 273 to $393^{\circ} \mathrm{K}$.

From the required experimental data and the chosen equation of state the hypothetical perfect gas entropy can be calculated in four ways because it is possible to substitute the Clapeyron equation for parts of the data.

${ }^{7}$ J. S. Koehler and D. M. Dennison, Phys. Rev. 57, 1006 (1940).

${ }^{8}$ A. Borden and E. F. Barker, J. Chem. Phys. 6, 553 (1938)

${ }^{9}$ D. G. Burkhard and D. M. Dennison (private communication). 
TABLE I. Entropy of translation, rotation, and vibration for methyl alcohol at one atmosphere.

\begin{tabular}{cccc}
\hline \hline$T\left({ }^{\circ} \mathrm{K}\right)$ & $S_{(t+r)}$ & $S$ (vib.) & $S^{\circ}-S_{r r}$ \\
\hline 273.2 & 54.581 & 0.138 & 54.719 \\
293.2 & 55.143 & 0.201 & 55.344 \\
313.2 & 55.667 & 0.278 & 55.945 \\
333.2 & 56.159 & 0.370 & 56.529 \\
353.2 & 56.623 & 0.478 & 57.101 \\
373.2 & 57.060 & 0.599 & 57.659 \\
393.2 & 57.475 & 0.729 & 58.304 \\
\hline
\end{tabular}

The usual calculation uses the experimental heat of vaporization, the vapor pressure and a correction based upon the equation of state for deviation from ideal gas behavior. The heat of vaporization, however, can be calculated from the vapor pressure and the equation of state, a new equation of state can be derived and used, or a new vapor pressure curve can be calculated from the thermal data and the equation of state, provided that one point on the curve is known or assumed. Thus it is possible to set up a trial barrier as a function of the temperature in four ways, and, by comparing the results, to show which data are probably in error and where the true barrier should lie.

If all of the data, including the equation of state, were accurate, the same constant barrier would be obtained at all temperatures by any of the alternative calculations. In this connection it is safe to assume that any anharmonicity effect such as that proposed by Scott, Waddington, Smith, and Huffman ${ }^{10}$ for the benzene case is negligible. It is necessary, however, to use the equation of state in the simplified form

$$
p V=R T+B p
$$

in which $B$ is a function of the temperature alone and is evaluated from data taken at constant pressure. If the expression for $B$ is used at other pressures an error will be introduced. The resulting entropy of gas imperfection, $p d B / d T$, should be nearly correct at low pressures, but will be low at high pressures, as the neglected terms in powers of the pressure become important. Consequently, trial barriers obtained with this form of the equation of state should increase with the temperature.

A qualitative idea of the effect can be obtained from the data for water vapor, for which the deviation entropy has been calculated ${ }^{\prime 1}$ from the virial coefficients tabulated by Keyes. ${ }^{12}$ The deviation volume at the saturation pressure is calculated from the same coefficients and the entropy effect is estimated as $p d B / d T$. The result is low to an increasing degree with increasing temperature. The effect for methyl alcohol, based upon a single isobar, should be in the same direction but of smaller magnitude. The trial barrier may then be expected to increase, slowly at first, but more rapidly

${ }^{10}$ Scott, Waddington, Smith, and Huffman, J. Chem. Phys. 15,565 (1947).

i1 J. O. Halford, J. Chem. Phys. 17, 405 (1949).

12 F. G. Keyes, J. Chem. Phys. 15, 611 (1947).
TABLE II. Entropy of methyl alcohol vapor at the saturation pressure.

\begin{tabular}{cccc}
\hline & Liquid & & \\
$T\left({ }^{\circ} \mathrm{K}\right)$ & $S-S_{288.2}$ & $\Delta H / T$ & $S T$ (vapor) $-S_{\text {a }}$ \\
\hline 273.2 & -1.625 & 33.646 & 62.281 \\
293.2 & -0.322 & 30.706 & 60.644 \\
313.2 & +0.959 & 28.009 & 59.228 \\
333.2 & 2.233 & 25.509 & 58.002 \\
353.2 & 3.511 & 23.167 & 56.938 \\
373.2 & 4.803 & 20.945 & 56.008 \\
393.2 & 6.117 & 18.816 & 55.193 \\
\hline
\end{tabular}

later, with increasing temperature. It is a fair presumption that, if the barrier calculated from a selection of data behaves in this manner, the value obtained at the lowest temperature is near to the correct one.

The sum of the statistical contributions to the perfect gas entropy from all sources except the internal rotation is first calculated. This is subsequently compared with the third law value obtained in several different ways to give the entropy of the internal rotation, which is then translated into the equivalent potential barrier by reference to the tables of Pitzer and Gwinn..$^{13}$ The physical constants used throughout the calculations are taken from a circular distributed by the National Bureau of Standards, bearing the date December 31, 1947.

For the rotational entropy of the rigid molecule the product of the principal moments of inertia is required, while the determination of the potential barrier uses the reduced internal moment of inertia. Burkhard and Dennison ${ }^{9}$ have evaluated the large principal moment $(A)$ and have obtained a close approximation to the moment of the $\mathrm{OH}$ group $\left(C_{1}\right)$ about an axis through the center of gravity parallel to the symmetry axis of the methyl group. The difference between them, $A-C_{1}=B$ is near to a second principal moment. The moment of inertia of the methyl group $\left(C_{2}\right)$ is taken from the spectroscopic value for the methyl halides. For the present purpose, the effect of the small product of inertia due to the off-center location of the hydroxyl hydrogen is neglected, and the moments are taken as $A=35.1\left(\times 10^{-40} \mathrm{~g} \mathrm{~cm}^{2}\right) B=34.1, C_{1}=1.01, C_{2}=5.50$, $C=C_{1}+C_{2}=6.51$, and, for the reduced internal moment $C_{1} C_{2} / C=0.845$.

The true moment product for the rigid molecule is slightly less than $A B C$. Also it is probably true that the ratio of two evaluations of Kassel's ${ }^{14}$ determinant, respectively with and without the internal rotation, should be used for the internal moment. This ratio is $0.815 \times 10^{-40}$, but the larger internal moment is used here because of its prior use in determining the potential barrier. The correct product of principal moments and the lower internal moment would each lead to lower values of the potential barrier.

For the translational and rotational entropy at one

\footnotetext{
${ }^{13}$ K. S. Pitzer and W. D. Gwinn, J. Chem. Phys. 10, 428 (1942).

${ }^{14}$ L. S. Kassel, J. Chem. Phys. 4, 276 (1936).
} 
atmosphere, the equation is

$S(t+r)=4.516+\left(\frac{3}{2}\right) R \ln M+\left(\frac{1}{2}\right) R \ln A B C+4 R \ln T$

in which $M$ is the molecular weight in grams and $A, B$ and $C$ are moments of inertia in $\mathrm{g} \mathrm{cm}^{2} \times 10^{38}$.

Vibrational frequencies have been assigned by Borden and Barker ${ }^{8}$ and by Noether. ${ }^{15}$ The differences introduced by Noether which are pertinent to the present calculation are the substitution, for two degrees of freedom, of the frequencies 1209 and 1260 for 1034 and 1340. The result is a small decrease of the vibrational entropy, relative to the Borden-Barker assignment, and a corresponding increase in the entropy available to the internal rotation. The effect of this increase upon the derived potential barrier is very small relative to other uncertainties. Noether's assignment has been used in the present calculations, merely because it is the later one, although it is not obvious that it is the correct one.

Table I shows, successively, for a range of temperatures, the entropy of translation and rotation, the vibrational entropy and their sum, $S^{0}-S_{r r}$, where $S_{r r}$ is the entropy of the restricted rotation. Any error in the last column due to the moments of inertia is constant, while the effect of a wrong frequency will increase with the temperature.

The third law entropy is based upon Kelley's ${ }^{16}$ value of $30.26 \pm 0.2 \mathrm{cal} . / \mathrm{mole}$ deg. for the liquid at $298.2^{\circ} \mathrm{K}$, which is combined with the heat capacity and heat of vaporization of Fiock, Ginnings, and Holton ${ }^{17}$ as shown in Table II. Any correction of Kelley's value called for by the newer physical constants can be neglected. The last column gives the entropy increase above that retained at the absolute zero, $S_{0}$.

The correction for deviation from perfect gas behavior is based upon the equation for the second virial coefficient proposed by Eucken and Meyer, ${ }^{18}$

$$
B(c c)=80-1.65 \times 10^{18} / T^{6} .
$$

The gas imperfection entropy in cc atmos./deg. mole is $9.90 \times 10^{18} p / T^{7}$. Table III shows the increase of the hypothetical perfect gas entropy above the residual entropy, $S_{0}$, at one atmosphere.

TABLE III. Hypothetical perfect gas entropy of methyl alcohol at one atmosphere. (cal/mole deg.)

\begin{tabular}{ccccc}
\hline \hline$T\left({ }^{\circ} \mathrm{K}\right)$ & $p$ (atmos. $)$ & $\left(\begin{array}{c}\left(S^{*}-S\right) \\
\text { and } M)\end{array}\right.$ & $R \ln p$ & $\left(S_{\left.T^{0}-S_{0}\right)}\right.$ \\
\hline 273.2 & 0.0389 & 0.082 & -6.452 & 55.911 \\
293.2 & 0.1251 & 0.161 & -4.131 & 56.674 \\
313.2 & 0.3427 & 0.283 & -2.128 & 57.383 \\
333.2 & 0.8255 & 0.434 & -0.381 & 58.055 \\
353.2 & 1.764 & 0.617 & +1.128 & 58.683 \\
373.2 & 3.451 & 0.821 & 2.461 & 59.290 \\
393.2 & 6.252 & 1.032 & 3.642 & 59.867 \\
\hline \hline
\end{tabular}

${ }^{15}$ H. D. Noether, J. Chem. Phys. 10, 693 (1942).

${ }^{16}$ K. K. Kelley, J. Am. Chem. Soc. 51, 181 (1929).

${ }^{17}$ Fiock, Ginnings, and Holton, J. Research Nat. Bur. Stand. 6, 881 (1931).

${ }_{18}$ A. Eucken and L. Meyer, Zeits. f. physik. Chemie 5B, 452 (1929).
It is unlikely that the vapor pressure will introduce a serious error. Consequently if a trial barrier has the predicted kind of temperature dependence, and the thermal data are accurate, it becomes probable that the deviation correction is accurate enough. This correction is independent of the constant term in Eq. (3).

The difference between the last columns of Tables I and III is $S_{r r}-S_{0}$. Table IV shows this quantity, together with two sets of trial barriers, for which, respectively, $S_{0}$ is assumed to be zero and 0.200 . The expression

$$
Q_{f}=2.7935\left(10^{38} I T\right)^{\frac{1}{2}} / 3
$$

has been used for the partition function of the limiting free rotator. The barriers are subject to error, particularly at the lower temperatures, because they require an extended extrapolation beyond the limits of the entropy table of Pitzer and Gwinn. The writer is fully aware of the factors which determined these limits and of the implications of such an extrapolation. However, for the purpose of seeing in a semi-quantitative way how nearly the entropy-based barrier can be brought to agreement with the spectroscopic one, the adopted procedure should be adequate. In the extrapolation, the attempt was made to choose relations between the variables which would lead to high, rather than low barriers. This difficulty will be removed by calculations, now in progress, of the thermodynamic properties directly from the newly determined molecular constants.

The predicted rise of the trial barriers at high temperatures appears in both sets. There is also a presumably false increase at the lower temperatures which could be due entirely or in part either to the extrapolation or to a fault in the experimental data. Each column shows a minimum, and it becomes probable that the true barrier would be somewhat less than the minimum obtained in a similar calculation with the correct residual entropy.

Further indications of the reliability of the data are now sought with the aid of the Clapeyron equation. The gas imperfection correction is taken, for the first check, from the heat of vaporization and the vapor pressure curve. The resulting trial barrier, for zero residual entropy, starts at 1300 and ends at 2300 after passing through a minimum near 600 , and a qualitatively similar situation would appear for other assumed residual entropies. If the heat data are correct, this

TABLE IV. Trial potential barriers for methyl alcohol (cal./mole deg.)

\begin{tabular}{cccccc}
\hline$T\left({ }^{\circ} \mathrm{K}\right)$ & $S_{r r}-S_{0}$ & $1 / Q f$ & $R T$ & $V\left(S_{0}=0\right)$ & $V\left(S_{0}=0.200\right)$ \\
\hline 273.2 & 1.192 & 0.7068 & 542.9 & 1730 & 1300 \\
293.2 & 1.330 & 0.6823 & 582.6 & 1625 & 1080 \\
313.2 & 1.438 & 0.6601 & 622.4 & 1630 & 1060 \\
333.2 & 1.526 & 0.6400 & 662.1 & 1610 & 1010 \\
353.2 & 1.582 & 0.6216 & 701.9 & 1650 & 1020 \\
373.2 & 1.631 & 0.6047 & 741.6 & 1710 & 1090 \\
393.2 & 1.663 & 0.5892 & 781.4 & 1830 & 1220 \\
\hline
\end{tabular}


effect must be due to errors in the vapor pressures. It is possible, however, for $d p / d T$ to be responsible without meaning that the pressures themselves are seriously wrong. The slope, in general, is too high, and, if the vapor pressure near the boiling point is assumed to be correct, it follows that the pressures are low at low temperatures and high at high temperatures. Correction for this effect would tend to decrease or eliminate the minima of Table IV, and would support the conclusion that the true barrier should be below the minimum one obtained with the data.

The next check omits the heat of vaporization and uses the vapor pressures and the equation of state. Here the trial barriers start at a low level and decrease with the temperature to the free rotation limit and beyond. The calculation follows the equation

$$
\begin{array}{r}
S^{0}-S_{l}=R T d \ln p / d T+\left(B-V_{l}\right) d p / d T \\
+R \ln p+p d B / d T,
\end{array}
$$

in which the term containing $V_{l}$ is negligible. The first term produces an effect in the observed direction, but could hardly be responsible for the extreme results obtained at higher temperatures. Consequently, since the last two terms have been used in preparing Table IV without coming under suspicion, it follows that the constant term in $B$ is mainly responsible for the high entropy. This constant becomes an increasingly important part of the deviation volume with increasing temperature.

A third check can be made by combining the equation of state, the heat of vaporization, the Clapeyron equation and a single vapor pressure at $333.2^{\circ} \mathrm{K}$, near the boiling point. The slope of the vapor pressure curve is calculated and new vapor pressures are found by graphical integration. They are not much different from the original vapor pressures and they lead to small decreases in the trial barriers at the lowest temperatures. These barriers are not reliable, however, because, as shown above, the constant term in $B$ is probably inaccurate. If this constant were adjusted to a more reasonable value, the required change in the vapor pressures and the trial barriers would be decreased.

From these checks upon the data no reason appears for changing the conclusion drawn directly from Table IV. The errors revealed by the analysis appear to involve principally $d p / d T$ and the constant term of Eq. (3), neither of which enters directly into the tabulated barrier calculation. The barrier estimate has also been made in the same manner for $C_{1}=1.4 \times 10^{-40}$, resulting in $V_{0}=2200 \pm 700$ cal. If it becomes necessary to revise $C_{1}$ to an intermediate value, it will be accurate enough to obtain the corresponding barrier by linear interpolation between the values given here.

French and Rasmussen ${ }^{3}$ obtained a barrier of 1350 cal./mole by selecting from the literature a heat of vaporization favorable to their empirical scheme for predicting potential barriers. If their calculation were repeated with the new moments of inertia the result would be close to the spectroscopic barrier. Their selected heat of vaporization, however, cannot be aligned with that of Fiock, Ginnings, and Holton ${ }^{17}$ at the boiling point to give an acceptable temperature variation of the calculated barrier. Since the Fiock, Ginnings, and Holton value at the boiling point checks earlier measurements of high reliability, it follows that the barrier calculation by French and Rasmussen is not a valid one. These authors have stated that the high barrier proposed by Crawford (3400) is due to the use of an older set of fundamental physical constants. Actually, the choice of constants could not affect the derived barrier by much more than 100 cal. Instead, the high result is due to an alternative relation between the entropy and the potential barrier proposed by Crawford. This relation has been disregarded in the present study because Pitzer and Gwinn ${ }^{13}$ have stated that their tables are accurate for all molecules described as rigid frameworks with one symmetrical top attached. The writer has studied their theory and method of calculation in enough detail to be convinced that their statement is correct.

From the last column of Table IV it can be concluded that the barrier derived from the entropy will be below $1000 \mathrm{cal}$. if an error or residual entropy of $0.20 \mathrm{cal} . / \mathrm{mole}$ deg. can be accounted for. Kelley's ${ }^{16}$ estimate of the uncertainty of the liquid entropy $( \pm 0.20)$, combined with other errors to which the calculation is subject, can account for an uncertainty of at least \pm 0.25 . It thus appears at the present writing that the spectroscopic barrier ( $932 \mathrm{cal}$.) lies near the lower limit of the range calculable from the entropy with the assumption that there is no retention of entropy by the crystal at the absolute zero. What has appeared heretofore to be a large and puzzling discrepancy becomes in these terms the result of an unfortunate combination of two large errors, one in the experimental data, the other in the molecular dimensions.

The barrier range derived from the entropy, 1600 $\pm 700 \mathrm{cal} . / \mathrm{mole}$, emphasizes that very accurate data are necessary for a barrier evaluation by this method. While the range includes the spectroscopic value, it also includes results corresponding to residual entropy at the absolute zero as high as $0.50 \mathrm{cal} . / \mathrm{mole} \mathrm{deg}$. In the light of Pauling's $\mathbf{s}^{19}$ discussion of hydroxy compounds, including water, which possess entropy due to the random orientation of hydrogen bonds, a similar situation appears probable for methyl alcohol and for other alcohols as well. The crystal could contain units of $n$ molecules arranged in chains or cycles which could assume two alternative forms, consistent with the presumption that each link between hydrogen and oxygen must be one of two kinds and that each atom of the $\mathrm{OH}$ groups is attached to its neighbors by two bonds, one of each kind. For this situation, if $n$ is a

${ }^{19}$ L. Pauling, J. Am. Chem. Soc. 57, 2680 (1935). 
fixed constant, the entropy retained in the crystal is $(1 / n) R \ln 2$ or $1.38 / n$. The maximum deficiency from the data is not far from the value for $n=3$, which, for a cyclic unit, would produce an arrangement exactly like one face of the tetrahedron of the ice structure.

This explanation appeals to the writer as a more probable one than the case for zero retention of entropy.

Quantitative consideration of the vapor heat capacity data will be deferred until this property has been accurately calculated from the molecular constants. The present required extrapolation of the table of Pitzer and $\mathrm{Gwinn}^{13}$ would introduce too large an uncertainty. Qualitatively, however, it is expected that the new dimensions will cause about the same decrease of the calculated barrier as in the case of the entropy. If this is true, the data of Eucken and Franck ${ }^{5}$ will lead to a barrier around $1200 \mathrm{cal} . / \mathrm{mole}$, that of Rowlinson ${ }^{6}$ to about $700 \mathrm{cal} . / \mathrm{mole}$.

\section{Note on Ethyl Alcohol}

In a recent analysis of the entropy of ethyl alcohol ${ }^{20}$ it was found that, for zero residual entropy, the sum of the barriers opposing the rotation of the methyl and hydroxyl groups was approximately $6000 \mathrm{cal} . / \mathrm{mole}$, possibly a little lower. If the situation is like the methyl alcohol case, the true sum could be almost as low as 4000 cal. The arbitrary assignment of 3000 to the methyl group would then leave 1000 for the hydroxyl group, exactly the value derived by Eucken and Franck $^{5}$ from the vapor heat capacity.

The writer is indebted to Professor D. M. Dennison and Dr. D. G. Burkhard for permission to use their data and for stimulating discussions of this problem and related questions.

${ }^{20}$ J. O. Halford, J. Chem. Phys. 17, 111 (1949),

\title{
On the Non-Orthogonality Problem Connected with the Use of Atomic Wave Functions in the Theory of Molecules and Crystals
}

\author{
Per-Olov LöwdIN \\ Institute of Mechanics and Mathematical Physics, University of Upsala, Trädgårdsgatan, Upsala, Sweden
}

(Received August 25, 1949)

\begin{abstract}
The treatment of molecules and crystals by the Heitler-London method or by the collective electron model can be based on the atomic orbitals $\phi_{\mu}$ of the system. These orbitals are in general overlapping, and the corresponding overlap integrals $S_{\mu \nu}$, given by (1), have almost universally been neglected in the literature as causing undesirable complications. Here we will take these overlap integrals into consideration and show that they, instead of being negligible, are of essential importance in molecules and in crystals. The problem is simply solved by considering the orthonormalized functions $\varphi_{\mu}$, given by (21), as the real atomic orbitals. The solution is worked out in detail for (I) the molecular orbital method of treating molecules, (II) the Bloch orbital method of treating crystals, and (III) the Heitler-London method of treating both these systems in some simple spin cases. Some numerical applications are given for ionic crystals, showing that the overlap effects are responsible for all the repulsive forces in these solids. It is also shown that the overlapping adds interesting new features to the properties of molecules and crystals, namely the "many-orbital-effects," corresponding to certain "many-body-forces" in ionic solids.
\end{abstract}

\section{INTRODUCTION}

$I^{\mathrm{N}}$ $\mathbf{N}$ treating the properties of molecules and crystals (metals, ionic solids, etc.) by means of quantum mechanics two principal methods have been developed: the Heitler-London method using atomic orbitals $(A O)$, and the collective electron model using molecular orbitals (MO) for the molecules and Bloch orbitals $(B O)$ for the crystals. Even in the latter case the $M O$ and the $B O$ could be constructed by "linear combination of atomic orbitals" ( $L C A O$ ), and hence the $A O$ can constitute the starting point in both methods.

For the sake of simplicity we assume from the beginning that the atomic nuclei in the molecules and crystals under consideration have fixed positions. This means that we confine ourselves to the case of absolute zero of temperature and neglect the zero-point vibrations. Each $A O$ is associated with an atomic nucleus and, if these nuclei are not too far from each other, the $A O$ are then overlapping.

Let $\phi_{\mu}(\mu=1,2, \cdots n)$ denote the complex or real atomic orbitals, which here are functions only of a space vector $\mathbf{r}$; the spin being excluded. They may be normalized, so that $\int \phi_{\mu}{ }^{*} \phi_{\mu} d \tau=1$, where $d \tau$ is the volume element, and the integration is to be carried out over the whole space. Then we define the "overlap integrals" by

$$
S_{\mu \nu}=\int \phi_{\mu}{ }^{*} \phi_{\nu} d \tau-\delta_{\mu \nu},
$$

with $S_{\mu \mu}=0$. These integrals are often small compared to unity, and, excepting some simple special cases, they have almost universally been neglected in the literature as causing undesirable complications. The existence of an overlapping problem has been pointed out by many 